\title{
INDUCTIVE LOGIC PROGRAMMING FOR INDUSTRIAL CONTROL APPLICATIONS
}

\author{
Samiya Bouarroudj ${ }^{1,2}$ and Zizette Boufaida ${ }^{2}$ \\ ${ }^{1}$ Department of Mathematics and Computer Science, \\ High School ENSET, Skikda, Algeria \\ ${ }^{2}$ LIRE Laboratory, Constantine 2 University, Constantine, Algeria \\ bouarroudjsamiadyahoo. fr ${ }^{1}$ \\ zboufaida@gmail. $\mathrm{com}^{2}$
}

\begin{abstract}
Advanced Monitoring Systems of the processes constitute a higher level to the systems of control and use specific techniques and methods. An important part of the task of supervision focuses on the detection and the diagnosis of various situations of faults which can affect the process. Methods of fault detection and diagnosis $(F D D)$ are different from the type of knowledge about the process that they require. They can be classified as data-driven, analytical, or knowledgebased approach. A collaborative FDD approach that combines the strengths of various heterogeneous FDD methods is able to maximize diagnostic performance. The new generation of knowledge-based systems or decision support systems needs to tap into knowledge that is both very broad, but specific to a domain, combining learning, structured representations of domain knowledge such as ontologies and reasoning tools. In this paper, we present a decisionaid tool in case of malfunction of high power industrial steam boiler. For this purpose an ontology was developed and considered as a prior conceptual knowledge in Inductive Logic Programming (ILP) for inducing diagnosis rules. The next step of the process concerns the inclusion of rules acquired by induction in the knowledge base as well as their exploitation for reasoning.
\end{abstract}

\section{KEYWORDS}

Inductive Logic Programming (ILP); SHIQ+log; Hybrid Reasoning; Semantic Web Technologies; Control System; Knowledge Management.

\section{INTRODUCTION}

The increasing demand in quality, safety, availability and cost optimization of industrial processes requires the use of Advanced Supervision Systems. Traditionally, this aspect of the supervision was under the responsibility of the human operators, possibly assisted by a set of sensors and detectors. Nevertheless this apparatus was set up to control the process and not for the detection and the diagnosis of faults.

Consequently, the development of new approaches is essential for a robust monitoring. In this context, many approaches are developed for fault detection and diagnosis (Cf. § 2.3). They include data-driven, analytical, and knowledge based approaches [1]. Methods of faults detection and diagnosis mentioned above have their strengths and weaknesses. Thus the combination of complementary methods is an effective way to achieve high performance.

Dhinaharan Nagamalai et al. (Eds) : CSE, DBDM, CCNET, AIFL, SCOM, CICS, CSIP- 2014

pp. 229-241, 2014. (C) CS \& IT-CSCP 2014

DOI : $10.5121 /$ csit.2014.4420 
Since supervision models are dependent on disparate information drawn from distributed sources, shared semantics based on a common ontology offers a way to develop these linkages. We address this critical need in this paper. Ontologies are a suitable formal representation able to convey this complex knowledge, but their use in learning algorithms is still a research issue.

Inference rules may be crafted by the domain expert as part of the ontology design, or automatically learned by machine learning techniques. We focus on this latter case as a generic component to easily adapt them to new domains. However, as opposed to previous approaches, learning takes place in the ontology language to produce deductive diagnosis rules which is possible with inductive logic programming (ILP).

We propose also a framework allowing the cohabitation of rules acquired by induction and the ontology as well as their exploitation for reasoning and researches.

The use of large steam boilers is quite common in industry due to their advantageous features [2][3]. However, such facilities are subject to several operating failures that could expose the system structural integrity to serious hazard and huge economic and human life losses. Early detection of such faults under operation is of great importance: it helps in reducing possible damage to equipments and productivity loss caused by (otherwise) unscheduled boiler shut-down, and also ensures safety operation of the systems.

The paper is organized as follows: initially, a discussion of current methods of fault diagnosis is presented. So, the proposed fault diagnosis system is developed. After discussing system structure, the main steps of the methodology designed are described in detail.

\section{APPLICATION FIELD}

In order to make natural gas practical and commercially viable to transport from one country to another, its volume has to be greatly reduced. To obtain maximum volume reduction, the gas has to be liquefied (condensed) by refrigeration to less than $-161{ }^{\circ} \mathrm{C}$. This process also requires very strict safety measures and precautions during all liquefaction stages, due to the flammable nature of the gas involved.

The LNG (Liquefied Natural Gas) plant (GL-1K complex) is located at $5 \mathrm{~km}$ east side of Skikda, Algeria. It has an area of 92 hectars and has been in production since the early 1970s. Gas is sourced from the Hassi R'mel fields, which also supply Arzew plants.

The plant, which is owned and operated by Sonatrach -owned oil and Gas Company -, had grown to six trains by the 1990s with the last of these commissioned in 1981. An LNG train is a liquefied natural gas plant's liquefaction and purification facility. Each LNG plant consists of one or more trains to compress natural gas into liquefied natural gas. A typical train consists of a compression area, propane condenser area, methane, and ethane areas.

All the trains received upgrades in the 1990s to bring them up to required specifications and the plant was capable of producing 7.68 million tons of LNG per year.

\subsection{Steam boiler description}

The industrial steam boiler, an ABB ALSTOM type, installed in the complex of natural gas liquefaction, generates a nominal steam capacity of 374 tons/h at superheated steam conditions of 73 bars and $487{ }^{\circ} \mathrm{C}$. It is composed of three main parts: the main feedwater line, the steam 
generator and the main superheated steam line. Figure 1 shows a schematic representation of the steam generator.

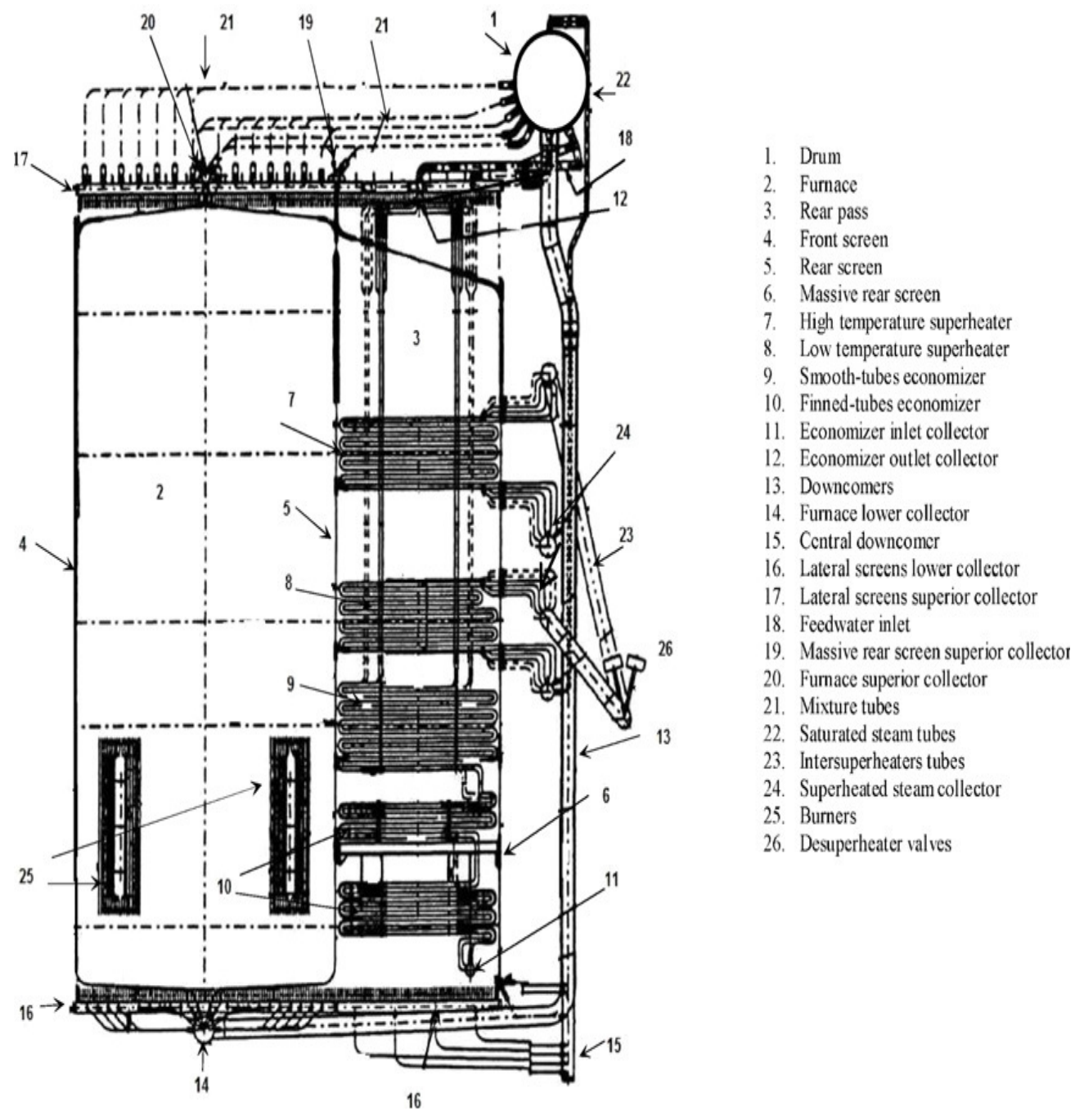

Figure 1. Cross-section view of steam generator

The integrated control and monitoring systems include various safety systems designed to prevent postulated damage during normal and abnormal transients. The top of the steam drum is equipped with three safety valves that control system pressure. Two other safety valves are installed on the main superheated steam line and there are four isolation valves and four flapper valves in different locations of the steam boiler facility.

\subsection{Problem of supervision}

In most chemical plants, distributed control systems (DCS) are used to simultaneously control thousands of process variables such as temperature and pressure. The main activities of plant operators in this control are to supervise these highly automated systems; fault detection and diagnosis, planning countermeasures; compensating and correcting abnormal situations. Operators are faced with these complex decision makings in managing abnormal situations. 
Monitoring manufacturing plants is a complex task. We are interested, in one hand, in monitoring and decision-aiding in case of malfunction of the production facility. In the other hand, when a malfunction occurs on the plant, this leads to some higher level problems. In fact, in such a case, we do not have any formal model of the plant, of the process and of the malfunction that occurred. For such high-risk production, we are interested in the more efficient way to handle a malfunction at the moment is to stop the production, which entails a financial impact. Our system allows extracting knowledge about the production facility during malfunction situations. Later, this knowledge may be used during a decision-aid step.

\subsection{Overview of existing approaches of fault detection and diagnosis}

For developing decision support systems in process control engineering, three solution approaches of data driven, analytical, and knowledge-based have been identified as follows:

\subsubsection{Data-driven Approach}

These approaches have been increasingly adopted for feature extraction from historical databases developed from process operations. The most popular data-driven process monitoring approaches include principal component analysis (PCA) [4], Fisher discriminant analysis and partial leastsquares analysis (PLS).

\subsubsection{Analytical Approach}

The analytical approach generally involves detailed mathematical models which use some measured input $\mathrm{u}$ and output $\mathrm{y}$, and generates features such as residuals $\mathrm{r}$, estimation parameter $\mathrm{p}$, and estimation state $\mathrm{x}$. Then, based on these values, fault detection and diagnosis can be performed by comparing the observed feature values with those associated with normal operating conditions either directly or after some transformations. Analytical methods can be categorized into the two common methods of parameter estimation and observer-based method [5].

\subsubsection{Knowledge-based Approach}

The artificial intelligence technologies which are associated with knowledge-based approaches and adopted for monitoring, control, and diagnosis in the industrial process include expert systems, fuzzy logic,...[6]

\subsubsection{Integrated approaches for monitoring, diagnosis and control of industrial processes}

The approaches described above are often combined in existing systems. Due to growing complexity of current systems, the integration of these approaches into an intelligent system requires a framework which coordinates communication among the different solution modules. Advanced Monitoring Systems of processes have been recognized by academia and industry as a vital research area, which many research programs and industrial projects were initiated to investigate. The most relevant ones are presented in the following.

CHEM-DSS (Decision Support System for Chemical/Petrochemical Manufacturing Processes) is an initiative of the European Community (EC) Intelligent Manufacturing Systems consortium in collaboration with Japan and Korea [7]. The aim of the CHEM-DSS project is to develop and implement an advanced Decision Support System (DSS) for process monitoring, data and event analysis, and operation support in industrial processes, mainly in refining, chemical and petrochemical processes. The research instead focused on analyzing the properties of the individual techniques of the system such as FDI, planning, artificial intelligence, signal 
processing, and scheduling. So, twenty three software toolboxes were developed during the project.

MAGIC (Multi-Agent-Based Diagnostic Data Acquisition and Management in Complex Systems) [8] is developed by a joint venture of several European universities and companies. The MAGIC system consists of several model-based and cause-effect diagnostic agents and a process specification agent to specify the process to be monitored and diagnosed. Depending on the process specifications, the appropriate data and knowledge acquisition is performed by another agent. A diagnostic decision agent and a diagnostic support agent propose a final diagnostic decision, which is displayed with other information to an operator interface agent. The MAGIC system prototype is developed for the metal processing industry.

However, knowledge of control systems mentioned above is not available in structured formats. For this reason, the new generation of decision support systems needs to tap into knowledge that is very broad combining learning, structured representations of domain knowledge such as ontologies and reasoning tools. It is in this context that joins our reflection.

\section{Inductive Logic Programming}

Inductive Logic Programming (ILP) [9] was born at the intersection of Concept Learning and Logic Programming. It is a supervised machine learning technique that uses logic programming as a representation for examples, background knowledge, and hypotheses. Given an encoding of the known background knowledge and a set of examples represented as a logical database of facts, an ILP system derives a hypothesized logic program which entails all the positive examples.

Though the use of background knowledge has been widely recognized as one of the strongest points of ILP when compared to other forms of Concept Learning, the background knowledge in ILP is often not organized around a well-formed conceptual model. This practice seems to ignore the growing demand for an ontological foundation of knowledge in intelligent systems. Rather, it highlights some difficulties in accommodating ontologies in ILP. Indeed the underlying Knowledge Representation (KR) frameworks (DLs (Description Logics) and HCL (Horn Clausal Logic) respectively) are deeply different in several respects but can be combined according to some limited forms of hybridization.

\subsection{Induction in ILP}

Induction in ILP generalizes valid hypotheses from individual instances/observations in presence of background knowledge. In Concept Learning, generalization is traditionally viewed as a search through a partially ordered space of inductive hypotheses [10]. According to this vision, an inductive hypothesis is a clausal theory and the induction of a single clause requires (1) structuring, (2) searching and (3) bounding the space of clauses [11].

\subsection{Ontologies and relational learning in ILP}

An ontology formally represents knowledge as a set of concepts of a specific domain and the relationships between these concepts. The widely accepted definition of ontology is "a formal, explicit specification of a shared conceptualization" [12].

Hybrid KR systems combining DLs and (fragments of) HCL have very recently attracted some attention in the ILP community. Three ILP frameworks have been proposed which adopt a hybrid 
DL-HCL representation for both hypotheses and background knowledge: Carin-ALN, resorts to AL-log, and builds upon SHIQ+log.

\subsubsection{Learning in Carin-ALN}

The framework proposed in [13] focuses on discriminant induction and adopts the ILP setting of learning from interpretations. Hypotheses are represented as CARIN-ALN non-recursive rules with a Horn literal in the head that plays the role of target concept. The coverage relation of hypotheses against examples adapts the usual one in learning from interpretations to the case of hybrid CARIN-ALN BK. The generality relation between two hypotheses is defined as an extension of generalized subsumption. Procedures for testing both the coverage relation and the generality relation are based on the existential entailment algorithm of CARIN.

\subsubsection{Learning in AL-log}

In [11], hypotheses are represented as constrained Datalog clauses that are linked, connected (or range-restricted), and compliant with the bias of Object Identity (OI). Therefore the literal in the head of hypotheses represents a concept to be either discriminated from others (discriminant induction) or characterized (characteristic induction). The generality relation for one such hypothesis language is an adaptation of generalized subsumption, named B-subsumption, to the AL-log KR framework. It gives raise to a quasi-order and can be checked with a decidable procedure based on constrained SLD-resolution.

\subsubsection{Learning in SHIQ+log}

This ILP framework represents hypotheses as SHIQ+log rules restricted to positive Datalog [14] and organizes them according to a generality ordering inspired by generalized subsumption. The resulting hypothesis space can be searched by means of refinement operators either top-down or bottom-up. A decidable KR framework SHIQ+log is the most powerful among the ones currently available for the integration of DLs and HCLs.

\section{ARCHITECTURE OF OUR SYSTEM}

Our system is developed to identify the causes and provides operation suggestions when abnormal situations occur. Figure 2 shows the main architecture of our system. This architecture consists of two distinct parts, one used offline which includes a module for generating examples and a module for learning discriminative patterns, and the other is used on line which includes a module for semantic reasoning.

\subsection{Steam boiler ontology construction}

Several ontologies have been proposed in the field of process engineering for the design and operation of chemical and pharmaceutical processes. Among the earliest, multi-dimensional formalism was developed as a collection of interrelated ontologies for describing the plant structure, the materials involved, the behavior of the material based on physico-chemical transformations occurring in the plant, and concepts to specify typical tasks in plant operation. This collection of ontologies was proposed to support engineering activities across the life cycle of the plant.

Development cost of these knowledge-based systems is often high because knowledge bases are often constructed from scratch in a distributed and heterogeneous environment. 
Relational databases are valuable sources for ontology learning. In this paper, we describe an approach for steam boiler ontology construction using heterogeneous databases. Our objective is to build an ontological resource, in a most automated way. The main data and information constituting our system come from disparate databases for equipment characteristics.

Methods and tools have been proposed to generate ontologies from such structured input. The mappings are the correspondences between each created ontology component (e.g., concept, property) and its original database schema concept (e.g., table, column).

The implementation of the proposed solution is realised using Protégé Plug-in DaTaMaster and it followed the steps below:

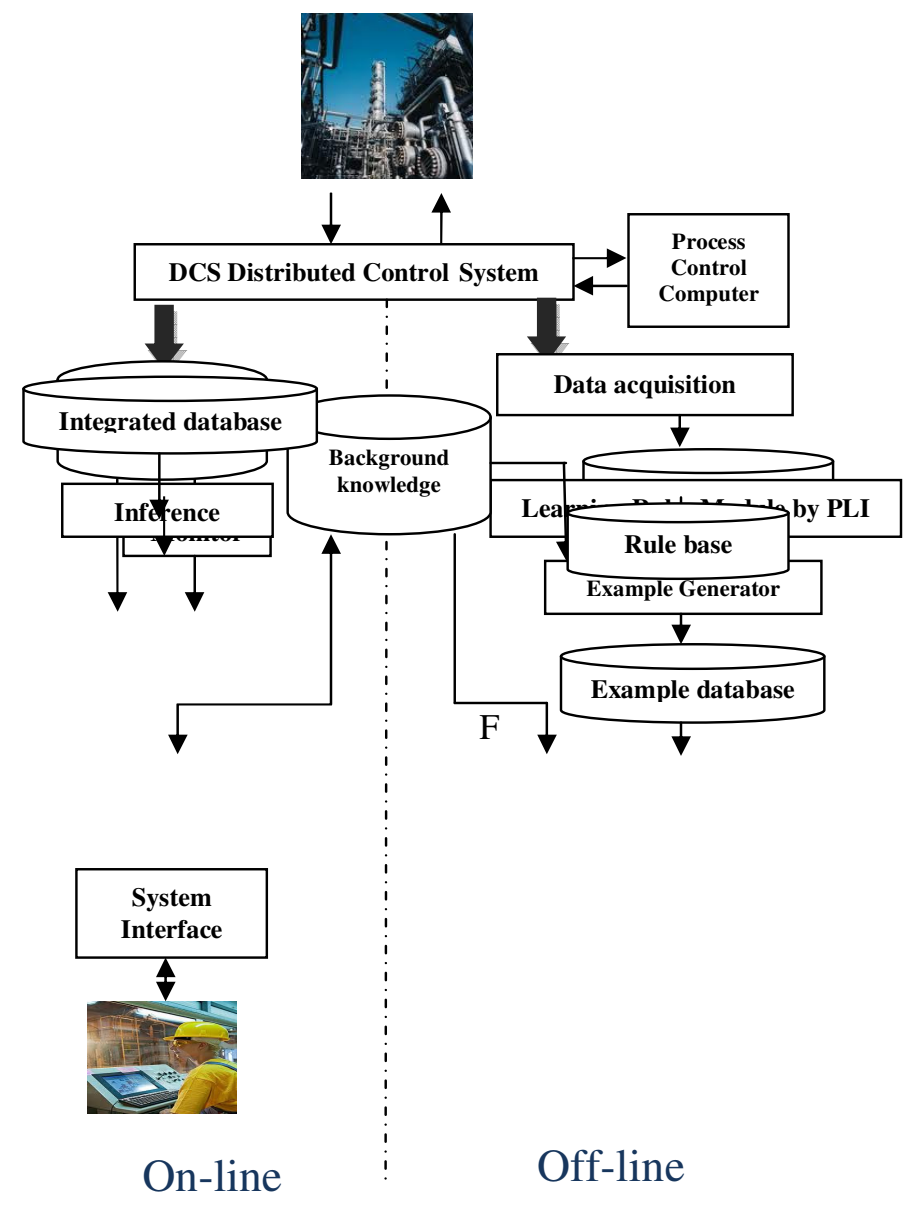

Figure 2. Architecture of the proposed system

- The choice of the connection driver types: Open Data Base Connectivity (ODBC) or Java Databases Connectivity (JDBC) and the data source.

- The selection of a given table activates the visualization of its content, then the user have the choice of importing the table or not.

- The chosen data base tables are activated and visualised, each table is transferred into one class or sub-class depending on the user's choice. 


\subsection{Distributed Control System (DCS)}

A Honeywell Distributed Control System (DCS) operates in the Production Unit, which serves the data via the so-called Process History Database (PHD) module. This database contains the most important process variables and some technological variables calculated by the Advanced Process Control (APC) module of the DCS.

\subsection{Data acquisition and Data Warehouse}

Large and complex industrial processes such as chemical plants and petroleum refineries are typically equipped with distributed control systems (DCS) which allow users to vary the number of alarms for the purpose of better monitoring process-variables. As such industrial processes increase in size, the volume of alarm information being presented to the operator also increase.

They not only may be thousands of individual alarms, nuisance alarms could also distract the operator's attention from more important problems. Process DataWarehouse is a data analysisdecision support and information process unit, which operates separately from the databases of the DCS. It is an information environment in contrast to the data transfer-oriented environment, which contains trusted, processed and collected data for historic data analysis. The data collected into DW directly provide input for different data mining, statistical tools, like classification, clustering, association rules, etc.

The first phase in the knowledge discovery process is the generation of simulated data sets and data preprocessing.

\subsubsection{Generation of simulated datasets}

It is observed that the injection of disturbances will cause changes in plant measurements, while the measurements outputs of the model under normal operation conditions remain unchanged. So the differences between the distributed and normal measurement outputs are used to generate discrete alarm data.

\subsubsection{Data preprocessing}

Alarm databases in a gas plant consist of records of time-stamped event logs. An example of raw gas plant data showing different fields is presented in Figure 3. As a minimum requirement, each record contains fields that store information such as a unique identifier for each alarm tag, time/date, alarm priorities, alarm description, alarm settings and the possible states of an alarm tag which include activation $[\mathrm{ALM}]$, return $[\mathrm{RTN}]$ and acknowledge $[\mathrm{ACK}]$ event types. We assume that a possible alarm sequence could be $\{[\mathrm{ALM}] \rightarrow[\mathrm{RTN}]\}$, or $\{[\mathrm{ALM}] \rightarrow[\mathrm{ACK}] \rightarrow[\mathrm{RTN}]\}$ butnot $\{[\mathrm{ALM}] \rightarrow[\mathrm{ACK}] \rightarrow[\mathrm{ALM}]\}$,or $\{[\mathrm{ALM}] \rightarrow[\mathrm{RTN}] \rightarrow[\mathrm{ACK}]\}$.

During the data preprocessing phase it is extremely important to carefully investigate and prepare alarm data since real plant data tends to be inconsistent, with errors, missing values, outliners and duplicate values. 


\begin{tabular}{|c|c|c|c|c|c|}
\hline \multirow[b]{2}{*}{$\begin{array}{l}\text { Time Stamp / } \\
\text { Unit ID }\end{array}$} & \multicolumn{5}{|c|}{ EVENT JOURNAL - PROCESS ALARMS } \\
\hline & $\begin{array}{l}\text { Point Name / } \\
\text { Point Description }\end{array}$ & $\begin{array}{l}\text { Tripped } \\
\text { Value }\end{array}$ & $\begin{array}{l}\text { Alarm Type/ } \\
\text { Alarm Priority }\end{array}$ & $\begin{array}{l}\text { Status/ } \\
\text { Event }\end{array}$ & $\begin{array}{l}\text { Source/ } \\
\text { Sequence of Erents Info. }\end{array}$ \\
\hline $\begin{array}{l}10 / 08 / 201221: 39: 07 \\
48\end{array}$ & $\begin{array}{l}\text { PM413 } \\
\text { M/A POMPE GRAIS }\end{array}$ & & $\begin{array}{l}\text { OFFNORM } \\
\text { HIGH }\end{array}$ & $\begin{array}{l}\mathrm{ACK} \\
31\end{array}$ & $\begin{array}{l}\text { P2-APP56 } \\
\text { D4801 } 3\end{array}$ \\
\hline $\begin{array}{l}\text { 10/08/2012 21:39:06 } \\
48\end{array}$ & $\begin{array}{l}\text { YHS455 } \\
\text { LOCAL / DISTANCE }\end{array}$ & & $\begin{array}{l}\text { OFFNORM } \\
\text { LOW }\end{array}$ & $\begin{array}{l}\text { RTN } \\
21\end{array}$ & $\begin{array}{l}\text { P2-APP56 } \\
\text { DISTANCE DGPL03 }\end{array}$ \\
\hline $\begin{array}{l}\text { 10/08/2012 21:39:01 } \\
48\end{array}$ & $\begin{array}{l}\text { PM413 } \\
\text { M/A POMPE GRAIS }\end{array}$ & & $\begin{array}{l}\text { OFFNORM } \\
\text { HIGH }\end{array}$ & $\begin{array}{l}\text { ALM } \\
21\end{array}$ & $\begin{array}{l}\text { P2-APP56 } \\
\text { ARRET D4801 }\end{array}$ \\
\hline $\begin{array}{l}10 / 08 / 201221: 38: 33 \\
48\end{array}$ & $\begin{array}{l}\text { CM413 } \\
\text { M/A COMPRES C413 }\end{array}$ & & $\begin{array}{l}\text { OFFNORM } \\
\text { HIGH }\end{array}$ & $\begin{array}{l}\text { RTN } \\
21\end{array}$ & $\begin{array}{l}\text { P2-APP56 } \\
\text { MARCHE DGPL03 }\end{array}$ \\
\hline $\begin{array}{l}\text { 10/08/2012 21:38:01 } \\
48\end{array}$ & $\begin{array}{l}\text { PM413 } \\
\text { M/A POMPE GRAIS }\end{array}$ & & $\begin{array}{l}\text { OFFNORM } \\
\text { HIGH }\end{array}$ & $\begin{array}{l}\text { RTN } \\
21\end{array}$ & $\begin{array}{l}\text { P2-APP56 } \\
\text { MARCHE D4801 }\end{array}$ \\
\hline $\begin{array}{l}10 / 08 / 201221: 20: 22 \\
50\end{array}$ & $\begin{array}{l}\text { PI455 } \\
\text { STOCKAGE TK-411 }\end{array}$ & 20.000 & $\begin{array}{l}\text { PVLO } \\
\text { LOW }\end{array}$ & $\begin{array}{l}\text { RTN } \\
21\end{array}$ & $\begin{array}{l}\text { P2-APP56 } \\
21.008 \text { D5001 }\end{array}$ \\
\hline $\begin{array}{l}10 / 08 / 201221: 02: 09 \\
10\end{array}$ & $\begin{array}{l}\text { FI5513 } \\
\text { DISTRIB VAP DEM 6-S-3303 }\end{array}$ & & $\begin{array}{l}\text { BADPV } \\
\text { HIGH }\end{array}$ & $\begin{array}{l}\text { RTN } \\
21\end{array}$ & P2-APP56 \\
\hline
\end{tabular}

Figure 3. An example of raw gas plant data showing different fields

\subsection{Generator of examples}

The simulation process associates to each breakdown situation a set of sequences of observations, building up a learning database. We recall that we are interested in cases of plant malfunctions. We want to model these unusual situations. Thus, the set of positive examples is made up of symbolic descriptions of the state of the plant that leads to a malfunction. We use the ontology as hypothesis language, and instances of this ontology as example language.

The generation process is an ontology population task. It lies in the acquisition from simulator of new extensional knowledge, i.e. ontology's instances. Each example of the base of the sequences alarms follows the following syntax: Example (I, C, O)

The parameter I is the identifier of example. We number the examples by integers from 1 to 200 . The parameter $\mathrm{C}$ is a list of class that the example belongs. As for the last parameter, the parameter $\mathrm{O}$ corresponds to the object describing the sequence. In our simplified example, the background knowledge is made up of a symbolic description of the working environment that is the components of the plant (Cf. § 4.5).

For instance a positive example is defined, for instance, in the following way:

Example (1, default (V4-1, TRC 317), sequence ([increase (V4-1, pressure)], [closed (V4-1)], [increase (V4-1, flow)], [after (V4-1, P1)]))

\subsection{Diagnosis rules learning by PLI}

The ILP unit allows generating general diagnosis rules that guide the operator in an efficient way to handle future malfunctions. The main objective of our work is the automatic learning of diagnosis rules. The examples used for this purpose are sequences of events obtained by simulation, each one of them being labeled according to the malfunction having caused it. The learning technique is based on the inductive logic programming.

We consider the problem of learning rules from ontologies and relational data. We assume that the predicate in the rule head represents a concept to be characterized (characteristic induction). The data are represented as a SHIQ+log knowledge base B where the intensional part K (i.e., the 
TBox $\mathrm{T}$ plus the set $\mathrm{R}$ of rules) plays the role of background knowledge and the extensional part (i.e., the ABox A plus the set $\mathrm{F}$ of facts) contributes to the definition of observations. Therefore ontologies may appear as input to the learning problem of interest. The observations are represented as a finite set of logical facts E. E could generally discomposed into the positive examples $\mathrm{E}^{+}$and the negative ones $\mathrm{E}^{-}$.

The background knowledge is supposed to be insufficient to explain the positive observations and the logical translation of this fact is: $\mathrm{B}=\mathrm{E}^{+}$but there is no contradiction with the negative knowledge: $\mathrm{BUE}^{-} \perp$.So an ILP machinery with input $\mathrm{E}$ and $\mathrm{B}$, will output a program $\mathrm{H}$ such that $\mathrm{B} \mathrm{U} \mathrm{H}=\mathrm{E}$. So $\mathrm{H}$ constitutes a kind of explanation of our observations $\mathrm{E}^{+}$. The language $\mathrm{L}$ of hypotheses must allow for the generation of SHIQ+log rules. More precisely, we consider defined clauses of the form: $p(\boldsymbol{X}) \leftarrow r_{1}\left(\boldsymbol{Y}_{1}\right), \ldots, r_{m}\left(\boldsymbol{Y}_{m}\right), s_{l}\left(\boldsymbol{Z}_{l}\right), \ldots, s_{k}\left(\boldsymbol{Z}_{k}\right)$. Where $\mathrm{m} \geq 0, \mathrm{k} \geq 0$, each $\mathrm{p}(\mathrm{X}), \mathrm{r}_{\mathrm{j}}\left(\mathrm{Y}_{\mathrm{j}}\right), \mathrm{s}_{\mathrm{l}}\left(\mathrm{Z}_{\mathrm{l}}\right)$ is an atom, and the literal $\mathrm{p}(\mathrm{X})$ in the head represents the target concept.

Figure 4 reports the main procedure of an algorithm analogously to FOIL [15] for learning ontorelational rules. The outer loop learns new rules one at a time, removing the positive examples covered by the latest rule before attempting to learn the next rule. The inner loop searches a second hypothesis space, consisting of conjunctions of literals, to find a conjunction that will form the body of the new rule.

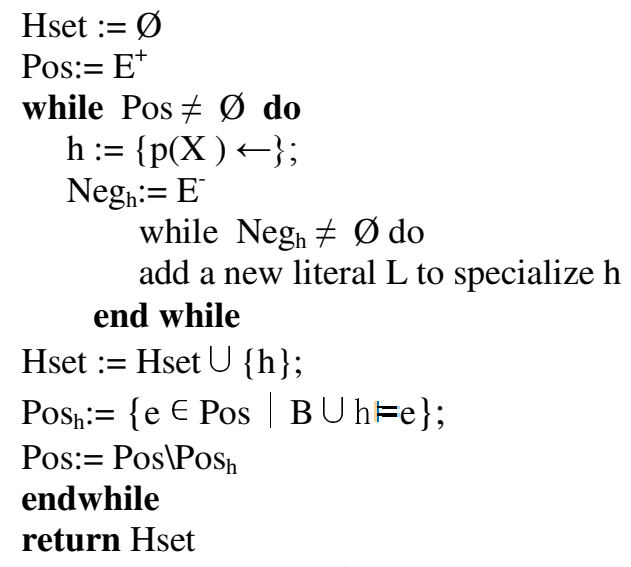

Figure 4. General algorithm for learning onto-relational rules

We want to show, on a small example, the way ILP may be used to induce a model of plant malfunctions. We want to model these unusual situations. Suppose we have a SHIQ+log KB consisting of the following intensional knowledge $\mathrm{K}$ :

Valve (C) $\sqsubseteq$ Component (C)

Pump (C) $\sqsubseteq$ Component (C)

Valves (v1)

........

Valve (v2)

Pump (P1)

Parameter (pressure)

We aim at inducing a definition for the predicate default/1 modeling the default of one component of the plant. In the entire system, the arity of this predicate may be greater than one depending on the number of components involved in the malfunction. If we run our system on the complete set of positive examples that describe the problem, the system induces the following definition for 
the predicate default/1: Default (A) $\leftarrow$ increase (A, pressure), after (A,P1), closed(A), which means that if the pressure increase in A and A is located after the pump P1 and A is closed then the component A causes a malfunction of the plant.

\subsection{Monitor}

The monitoring module is used to supervise production process. It monitors data streams obtained from the control system, e.g. temperature, pressure, and flow. If a situation is judged to be abnormal by the module, the data are automatically transferred to the inference machine to solve the problem. At the same time the data are stored in the integrated database.

\subsection{Inference machine}

It controls and executes problem solving. Semantics search is based on the application of reasoning techniques to perform logical deductions. This one results from raisoners able to use the semantics of OWL ontology. Regarding the deficiency of this language, our ontology is extended by diagnosis rules to increase its expressiveness. These rules represent the deductive part of a domain. They permit the propagation of relations and deduce new facts starting from the existing ones.

A hybrid reasoning mechanism developed in our previous works [16], is applied in this system of supervision. It ensures the reasoning and searches the rules efficiently. We suggest a combination method of reasoning to improve the search of results. The method is based on the principle of hybrid combination of two reasoners in which each treats a distinct party of knowledge base: a logical description raisoner for the structural part (OWL-DL) and a rule engine for the deductive part (RULES).

\subsection{Explanation instrument}

Tracks the route of reasoning and explains the results of the reasoning of system to the user upon request.

\subsection{Integrated database}

Stores facts, intermediate results of reasoning processes, real-time data, and historical data from the distributed computer system installed in the plant.

\section{CONCLUSION}

Complex processes involve many process variables, and operators faced with the tasks of monitoring, control, and diagnosis of these processes. They often find it difficult to effectively monitor the process data, analyze current states, detect and diagnose process anomalies, or take appropriate actions to control the processes.

To assist plant operators, decision support systems that incorporate artificial intelligence (AI) and non-AI technologies have been adopted for the tasks of monitoring, control, and diagnosis. In this paper, a real-time system is proposed for monitoring and diagnosing of chemical processes. The representation of knowledge base, inference machine and the relations among them are considered in this paper according to the characteristics of chemical processes. 
This system helps the operators a lot to eliminate potential and disasters faults. The system also decreases the loss brought by unstable process situations and the loss if the time used for eliminating faults is too long. When new fault occurs, the stored data helps the domain expert to analyze the reason of the fault, and give earlier prediction of the trend. Our system design approach can be exploited to develop and rapidly prototype real time distributed multi-agent systems.

\section{ACKNOWLEDGEMENTS}

The authors are very grateful toward all the personnel of the natural gas liquefaction complex of Skikda for providing all information related to the steam boiler equipments enabling the present study to be achieved.

\section{REFERENCES}

[1] H.L Chiang, L.E. Russell and D.R. Braatz, (2001) "Fault Detection and Diagnosis in Industrial Systems". Springer, London, Great Britain.

[2] Technical operations manual of the industrial steam boiler ABB ALSTOM (2000).

[3] D. R. Tucakovic, V. D. Stevanovic and T. Zivanovic, (2007) "Thermal hydraulic analysis of a steam boiler with rifled evaporating tubes." Applied Thermal Engineering, 27, 509-519.

[4] S. Yoon, F.J. MacGregor, (2004) "Principle-component analysis of multiscale data for process monitoring and fault diagnosis." AIChE Journal 50 (11), 2891-2903

[5] A.E. Garcia, M.P. Frank, (1996) "On the relationship between observer and parameter identification based approaches to fault detection." In: Proceedings of the 13th IFAC World Congress, vol. N. Piscataway., New Jersey, pp. 25-29.

[6] X. Luo, C. Zhang and R.N. Jennings, (2002) "A hybrid model for sharing information between fuzzy, uncertain and default reasoning models in multi-agent systems". International Journal of Uncertainty, Fuzziness and Knowledge-Based Systems 10 (4), 401-450.

[7] S. Cauvin, (2004) "CHEM-DSS : Advanced decision support system for chemical/petrochemical industry”, Fifteenth International Workshop on Principles of Diagnosis (DX'04), AAAI, Carcassonne, France

[8] B. Kőppen-Seliger, T. Marcu, M. Capobianco, S. Gentil, M. Albert, and S. Latzel, (2003) "MAGIC: An integrated approach for diagnostic data management and operator support," in Proceedings of the 5th IFAC Symposium Fault Detection, Supervision and Safety of Technical Processes SAFEPROCESS05, Washington D.C.

[9] S.Muggleton, (1999) "Inductive Logic Programming: Issues, Results and the Challenge of Learning Language in Logic", Artificial Intelligence, 114(1-2), pp.283--296.

[10] T. Mitchell, (1982) "Generalization as search. Artificial Intelligence" 18, 203-226.

[11] F. Lisi, (2008) "Building Rules on Top of Ontologies for the Semantic Web with Inductive Logic Programming.", Theory and Practice of Logic Programming 8(03), 271-300.

[12] A. Borgida, (1996) "On the relative expressiveness of description logics and predicate logics.", Artificial Intelligence 82(1-2), 353-367.

[13] C. Rouveirol, V. Ventos, (2000) “Towards Learning in CARIN-ALN.” In: Cussens, J., Frisch, A.M. (eds.) ILP 2000. LNCS (LNAI), vol. 1866, pp. 191-208. Springer, Heidelberg.

[14] F. Lisi, F. Esposito,(2007) "Building Rules on top of Ontologies? Inductive Logic Programming can help!" SWAP 2007

[15] J.R. Quinlan, (1990) "Learning logical definitions from relations.” Machine Learning, 5:239-266.

[16] S. Bouarroudj, Z. Boufaida, (2010) " A multi-reasoner system for semantic search of annotated images“, EGC-M 2010, Algiers , Algéria, pp. 118-129. 


\section{Authors}

Samiya BOUARROUDJ is a doctoral student of Computer Science at University of Constantine 2, Algeria. Her current research activities are conducted at the LIRE Laboratory. Here research interests are knowledge representation and reasoning, semantic web technologies and advanced monitoring systems of the processes.

Zizette BOUFAIDA is a Professor of Computer Science at University of Constantine 2, Algeria and the cohead of the SI\&BC research group at the LIRE laboratory. Her research interests include knowledge representation and reasoning; formal knowledge representation for Semantic Web, ontology development,... 